Rev. Q'EUÑA 10 (1):37- 47 (Agosto 2019)

ISSN: 2708-2660 (Ele), 2412-2297 (Imp)

Recibido: Enero 10, 2019

Sociedad Botánica del Cusco

Aceptado: Julio 22, 2019

\title{
Diversidad y composición de helechos arbóreos en la localidad de Wiñaywayna- Intipunku, Santuario Histórico de Machu Picchu
}

\section{Diversity and composition of tree ferns in Wiñaywayna - Intipunku locality, Historic Sanctuary of Machu Picchu}

\author{
María E. Holgado-Rojas ${ }^{1,2}$, Gloria Calatayud Hermoza ${ }^{1,2}$, Christian Alvarez Conza \& Hugo \\ Ccopa Huayta . \\ 'Universidad Nacional de San Antonio Abad del Cusco, Facultad de Ciencias, Escuela \\ Profesional de Biología. \\ 2Sociedad Botánica del Cusco. calatayudhglor@gmail.com
}

\section{Resumen}

Los bosques montanos Neotropicales albergan a la flora de helechos arbóreos más ricos del mundo, este es un grupo bastante conspicuo por su tamaño y frecuencia, sin embargo, poco estudiado y conocido. La familia muestra una distribución pantropical con más de 600 especies. Todas las especies de helechos arbóreos están incluidos en el Apéndice II de la Convención sobre el Comercio Internacional de Especies Amenazadas de Fauna y Flora Silvestres (CITES), por lo que consideramos necesario evaluar la diversidad y parámetros poblacionales de las especies presentes en un sector Wiñaywayna- Intipunku del Santuario Histórico de Machu Picchu (SHMP), Cusco, Sureste de Perú. La evaluación se en un transecto de 500 x 5 $\mathrm{m}$, dividido en sub unidades de $25 \times 5 \mathrm{~m}$, donde registramos 12 especies de helechos arbóreos de los géneros Cyathea, Dicksonia y Lophosoria. La especie con mayor índice de valor de importancia (IVI) fue $D$. sellowiana Hook. Estos resultados servirán como herramienta para guiar esfuerzos de conservación en los bosques montanos del SHMP.

Palabras clave. Diversidad, Helechos arbóreos, Parámetros poblacionales, Wiñay wayna-Intipunku.

\begin{abstract}
Neotropical mountain forest include the richest flora of tree ferns in the world. They are such a conspicuous group because of their size and frequency; however, they are little studied and known. Tree Ferns Family have pantropical distribution with more than 600 species. All species of tree ferns are included in the appendix of Convention on International Trade of Endangered Species of Wild Fauna and Flora (CITES) which is why we considered (it) necessary to evaluate the diversity and population parameters of the present species at a sector of the (a) Historical Sanctuary of Machu Picchu (HSMP) (sector). We sampled one line transect of $500 \times 5 \mathrm{~m}$, divided into (lots) transects of $25 \times 5 \mathrm{~m}$ in the Wiñaywayna -Intipunku zone, province of the Urubamba, Cusco region at $2653 \mathrm{~m}$. Results show a richness of 12 species of Cyathea, Dicksonia and Lophosoria. The species with the highest abundance (50.76\%) and dominance (84.36\%) was Dicksonia sellowiana followed by L.quadripinnata con 59.46 de IVI. These results will serve as a tool to guide conservation efforts in the SHMP mountain forests.
\end{abstract}

Key words. Diversity, Population parameters, Tree ferns, Wiñay wayna-Intipunku. 


\section{Introducción}

Las Pteridofitas fueron las primeras plantas vasculares que poblaron la tierra. Según el registro fósil apareció en el Silúrico hace 350 millones de años. Los helechos arbóreos llegaron a alcanzar 30 metros de altura, dominaron la tierra hace 320 millones de años, encontrándose la mayoría de estos en la familia Cyatheaceae (Rojas 1999).

Los helechos arborescentes son el componente más abundante en los bosques húmedos montanos tropicales y algunos bosques temperados del sur (RamírezBarahona et al., 2011, 2016). Los bosques montanos de los andes tropicales tienen una de las más ricas floras en el planeta con más de 200 especies descritas para este bioma y una alta proporción ocurre en Perú (Lehnert 2011).

El Santuario Histórico de Machu Picchu (SHMP) presenta variadas condiciones medioambientales que ha permitido la generación de una flora muy diversa, con exuberantes bosques de neblina que cubren las abruptas laderas y montañas con innumerables especies de flora silvestre, entre las que destacan delicadas orquídeas, bromelias y helechos arborescentes de gran importancia tanto ecológica como económica, actualmente se les considera como indicadores biológicos de lugares poco o nada perturbados, afirma Galiano et al. (2002).

Los helechos arbóreos junto con las orquídeas y cactáceas, constituyen uno de los principales grupos de plantas ornamentales, lo que ha provocado que algunas poblaciones naturales se vean amenazadas o en peligro de desaparecer.

El comercio ilegal ha impulsado su sobreexplotación. Además, socialmente se puede mencionar que estas plantas forman parte importante de la amplia gama de- -medicina tradicional de la región por lo que actualmente están incluidas en el apéndice II de la Convención sobre Comercio Internacional de Especies Amenazadas de Fauna y Flora Silvestre (CITES) (fig.07).

El propósito de este estudio fue analizar los parámetros poblacionales y la diversidad obtenidos de las especies presentes en el SHMP, sector Intipunku, servirán para respaldar con sustento científico las decisiones y criterios a tomar en cuenta para impulsar iniciativas locales de conservación y uso sostenible y garantizar su supervivencia.

\section{Área de estudio}

La parcela fue instalada en la zona de amortiguamiento del SHMP en la localidad de Wiñaywayna-Intipunku, provincia de Urubamba, región Cusco, en las coordenadas 767238 S, $8539086 \mathrm{~W}$, a una altura de $2653 \mathrm{~m}$. El SHMP se encuentra situado en una región correspondiente a la Selva Alta o Ceja de Selva. Es un área con gran heterogeneidad ambiental, que junto con su abrupto relieve orográfico y su régimen climático, hacen posible la existencia de numerosas zonas de vida, de acuerdo con el sistema de clasificación de Holdridge (1967) entre los 2000 y $6000 \mathrm{~m}$.

La ecología del SHMP es sumamente diversa y compleja, ya que incluye diez zonas de vida, desde el bosque seco montano bajo -a orillas del valle- hasta el nivel en las cumbres de la cordillera. En términos altitudinales, esto significa que se extiende desde los 1,725 m a nivel del río Urubamba, hasta los 6,271 m en la cumbre del nevado Salcantay. Estas variaciones geográficas, y su singular topografía, tienen por consecuencia una diversidad muy amplia de flora y fauna silvestres, afirma Galiano et al. (2002). 


\section{Métodos}

El registro y el muestreo de plantas se hizo dentro de un transecto de 500 x 5 m., se siguió el protocolo para el muestreo de helechos preparado por el Smithsonian Conservation Biology Institute - Center for Conservation Education and Sustainability como indica Tuomisto (2002). El transecto fue dividido en sub unidades de $25 \times 5 \mathrm{~m}$. Se usó una trocha, las estacas marcadas y un pabilo extendido para ubicar la línea central del transecto. Se registraron todos los helechos arbóreos dentro una faja de 2,5 m contados desde la línea central a ambos lados.

Se incluyeron sólo los helechos que presentaron por lo menos una hoja mayor o igual a $10 \mathrm{~cm}$ de longitud. El número de individuos por especie, la altura y otras características del individuo se registraron y se elaboró una base de datos. De los helechos más abundantes y fáciles de identificar solo se colectó un individuo para garantizar la identificación de la especie. Sólo los helechos arbóreos que se tuvo dificultad en la identificación en campo fueron colectados para su posterior determinación. No se encontró especímenes fértiles.

\section{Índices}

Para cuantificar el valor de diversidad y la estructura del área evaluada usamos los siguientes índices:

Riqueza específica (S): Corresponde al número total de especies obtenido por un censo de la comunidad.

Índice de diversidad de Margalef.

$\mathrm{D}_{\mathrm{mg}}=\underline{\mathrm{S}-1 / \ln (\mathrm{N})}$

Donde: $\mathrm{S}=$ Número total de especies y $\mathrm{N}=$ Número total de individuos.
Transforma el número de especies por muestra a una proporción a la cual las especies son añadidas por expansión de la muestra. Supone que hay una relación funcional entre el número de especies y el número total de individuos. El valor máximo se encuentra por medio de $(S-1) / \ln (N)$.Mientras más alto es el valor, más diversa es la muestra analizada. La desventaja de este índice es que es insensible a la igualdad de individuos por especie.

\section{Índice de dominancia de Simpson} (D), éste índice cuantifica la probabilidad que dos individuos seleccionados en una comunidad infinita pertenezcan a una misma especie. La probabilidad promedio de que ocurra será igual a la suma de las probabilidades individuales de cada especie. La fórmula resultante será:

$$
\mathrm{D}=\sum \mathbf{p}_{\mathrm{i}}{ }^{2} \quad \mathbf{p}_{\mathrm{i}}=\mathbf{n}_{\mathrm{i}} / \mathbf{N}
$$

Donde: Ni: representa la abundancia de la especie i, N: el número total de individuos en toda la comunidad.

Los valores de la diversidad según Simpson se dan dentro de una escala de 0 a 1 ; siendo mayor cuando se aproxima a uno y menor al acercarse a cero, lo que indicaría mayor dominancia.

La abundancia relativa por hábitat, corresponde al número de individuos en cada hábitat muestreado (Tejedor, 2013): Para determinar la abundancia relativa total por localidad se sumarán los índices de cada hábitat (Tab.1). 
Tabla 1:Abundancia relativa por hábitat

\begin{tabular}{|l|l|l|}
\hline Categoría & Escala & $\mathbf{N}^{\circ}$ Individuos \\
\hline Raro & 1 & $1-2$ individuos \\
\hline Infrecuente & 2 & $3-10$ individuos \\
\hline Frecuente & 3 & $11-20$ individuos \\
\hline Común & 4 & $21-40$ individuos \\
\hline Abundante & 5 & $>40$ individuos \\
\hline
\end{tabular}

El Índice de equidad de ShannonWiener $\left(\mathbf{H}^{\prime}\right)$, Es la medida del grado de incertidumbre que existe para predecir la especie a la cual pertenece un individuo extraído aleatoriamente de la comunidad. Los valores que se obtiene con este índice generalmente están entre 1 y raramente sobrepasa a 4.5, puede llegar 5 como señala Krebs (1989).

$$
\mathrm{H}=\sum=(\mathrm{pi})(\log 2 p i)
$$

Donde: $\mathrm{H}^{\prime}=$ Índice de Diversidad de Especies y $\mathrm{Pi}=$ Proporción del número de individuos de la especie i, con respecto al total (ni/Ni).

El máximo valor del índice de Shannon -Wiener para un número determinado de especies se puede calcular de la siguiente manera:

$$
H_{\text {max }}^{\prime}=\ln S
$$

Donde: $\mathrm{H}^{\prime}=$ Diversidad bajo condiciones de máxima equitatividad y $\mathrm{S}=$ Número de especies.

\section{El Índice de Valor de Importancia} (IVI), muestra la importancia ecológica relativa de cada especie en el área muestreada, interpreta a las especies que están mejor adaptadas, ya sea- -porque son dominantes, muy abundantes o cuenten con una distribución frecuente como dice Mostacedo (2000). Este valor nos permite tomar decisiones o emitir recomendaciones a favor de la conservación de la taxa o áreas amenazadas.

El IVI resulta de la suma de los valores relativos de frecuencia, densidad y dominancia (área basal). Los valores relativos alcanzan de 0 a 100, con la consecuencia de que los valores de importancia tanto de especies como de familias alcanzan de 0 a 300. Para facilitar la comparación de los datos calculados también se presentan los valores de importancia en porcentajes como indica Matteucci y Colma (1982).

Variables poblacionales: Acorde a lo que indica Matteucci y Colma (1982) tenemos:

Frecuencia (F): Se refiere al número de muestras (transectos) en el que una especie está presente, en referencia al total de unidades muestrales.

$$
\mathbf{F}=\mathbf{m i} / \mathbf{M}
$$

Donde: $\mathbf{m i}=$ Número de unidades muéstrales en las que la especie ocurre y $\mathbf{M}=$ Número total de unidades muéstrales

Frecuencia relativa (FR): Esta dada por la frecuencia total de una especie en referencia a la frecuencia total de todas las especies.

$\mathbf{F R}=$ (Frecuencia de una especie/suma de todas las frecuencias) x 100 
Densidad (D): Está definida como el número de organismos de una determinada especie por unidad de área y la Densidad relativa (Dr): Esta dada por la densidad total de una especie en referencia a la densidad total de todas las especies y Dominancia relativa: La relación expresada en porcentaje entre la dominancia absoluta de una especie cualquiera y el total de las dominancias absolutas de las especies consideradas en el área inventariada.

Dominancia. - Es la sumatoria del área basal de todos los individuos de una especie, por ésta razón se utilizó las áreas basales, además que existe una correlación lineal entre el diámetro de la copa y el fuste.

Área basal. - Según Matteucci y Colma (1982) "El área basal es la superficie de una sección transversal del tallo o tronco de un árbol a una determinada altura del suelo. En árboles, este parámetro se mide obteniendo el diámetro o el perímetro a la altura del pecho (DAP a una altura de $1.3 \mathrm{~m}$ ). En arbustos u otras plantas, que se ramifican desde la base, el diámetro o perímetro se toma a la altura del suelo”.

Donde: $\mathrm{DAP}=$ diámetro a la altura del pecho y $\mathrm{pi}=3.1416$

\section{Resultados}

\section{Riqueza y Diversidad}

Registramos un total de 65 individuos, pertenecientes a 12 especies de helechos arborescentes: C. carolihenrici, C. catacampta, C. delgadii, C. ruiziana, C. squamipes, Cyathea sp 1, Cyathea sp2, Cyathea sp3, Cyathea sp4, D. sellowiana, L.quadripinnata y C. conjugata (fuera- -del transecto de estudio). Dos especies fueron las más representativas, $D$ sellowiana y $L$. quadripinnata con $33(50.76 \%)$ y 15 (23.07\%) individuos respectivamente.

El índice de Margalef (1951); obteniendo un valor de 2.39 que refleja una riqueza media de especies (Tab. 2).

Los análisis para la estimación de la diversidad, muestran una diversidad baja según el índice de Shannon $\left(\mathrm{H}^{\prime}=1.57\right)$, y una diversidad media según el índice de Simpson $(1-\mathrm{D}=0.67)$ como indica la Tabla 2.

Los análisis nos muestran una diversidad baja según el índice de Shannon $\left(\mathrm{H}^{\prime}=1.57\right)$ y el índice de Dominancia de Simpson nos indicaría que tiene una diversidad media $(1-\mathrm{D}=0.67)$.

\section{Estructura:}

\section{Abundancia relativa por hábitat:}

D. sellowiana es una especie común en la zona de estudios, No obstante, siete especies son raras y dos infrecuentes. (Tab. 2).

Frecuencia (F): La frecuencia es la misma en todas las especies. Porque se realizó un sólo transecto de 500x 5m (Tab. 2).

Densidad (D): Como indica la Tabla 6, las especies con mayor densidad respecto al resto de especies (Dr) son D. sellowiana (50.76) y L. quadripinnata (23.07).

Dominancia: En la zona evaluada la mayoría de especies presentaron valores bajos de dominancia relativa, mientras que solo una sobresalió por la alta concentración de individuos. Siendo este D. sellowiana acumulando el $83 \%$ de la dominancia, como se muestra en la tabla 2. 


\section{Índice deValor de Importancia (IVI)}

Como indica la Tabla 2, la especie con mayor valor de importancia es D. sellowiana (143.7\%), seguida de L. quadripinnata (36.7\%) y las especies con menor importancia ecológica son C. carolihenrici (10.7) y Cyathea sp3 (10.6\%). (Tab. 2).
C. tungurague y C. cystolepis. Debemos aclarar que $C$. caracasana, es una especie que tiene una distribución a partir de Colombia, Venezuela, República Dominicana, Jamaica, Puerto Rico y Cuba (Lehnert, 2009).

Es decir que la especie $C$. caracasana reportada por Galiano y Nuñez (2011) podría ser-

Tabla 2. Índice de valor de Importancia de helechos arbóreos - sector Wiñaywayna-Intipunku

\begin{tabular}{|c|c|c|c|c|c|c|}
\hline $\mathbf{N}^{\circ}$ & Especie & ni & $\begin{array}{l}\text { Densidad } \\
\text { Relativa }\end{array}$ & $\begin{array}{l}\text { Frecuen } \\
\text { cia } \\
\text { Relativa }\end{array}$ & $\begin{array}{l}\text { Domin } \\
\text { ancia } \\
\text { Relativ } \\
\text { a }\end{array}$ & IVI \\
\hline 1 & Cyathea carolihenrici Lehnert & 1 & 1.54 & 9.09 & 0.11 & 10.74 \\
\hline 2 & Cyathea catacampta Klotzsch & 1 & 1.54 & 9.09 & 0.60 & 11.23 \\
\hline 3 & Cyathea delgadii Sternb. aff. & 3 & 4.62 & 9.09 & 2.92 & 16.63 \\
\hline 4 & Cyathea ruiziana Klotzsch & 2 & 3.08 & 9.09 & 5.58 & 17.75 \\
\hline 5 & Cyathea sp1 & 4 & 6.15 & 9.09 & 0.38 & 15.62 \\
\hline 6 & Cyathea sp2 & 2 & 3.08 & 9.09 & 0.89 & 13.05 \\
\hline 7 & Cyathea sp3 & 1 & 1.54 & 9.09 & 0.05 & 10.68 \\
\hline 8 & Cyathea sp4 & 1 & 1.54 & 9.09 & 0.99 & 11.62 \\
\hline 9 & Cyathea squamipes H. Karst. & 2 & 3.08 & 9.09 & 0.03 & 12.20 \\
\hline 10 & Dicksonia sellowiana Hook. & 33 & 50.77 & 9.09 & 83.85 & $\overline{143.71}$ \\
\hline \multirow[t]{2}{*}{11} & Lophosoria quadripinnata (J.F. Gmel.) C. Chr. & 15 & 23.08 & 9.09 & 4.62 & 36.78 \\
\hline & Total & 65 & 100.00 & 100.00 & 100.00 & 300.00 \\
\hline
\end{tabular}

\section{Discusiones}

\section{Riqueza y Diversidad}

Para el sector de Wiñaywayna-Intipuncu registramos 12 especies de helechos arbóreos. Hace algunos años, Galiano y Nuñez (2011) para el SHMP reportan 15 especies (Tab. 3). Sin embargo, C. erinacea H. Karst., sinónimo de A. erinacea (H. Karst.) D.S. Conant, es un nombre desactualizado. C.erinacea, es sinónimo de A. erinacea (H. Karst.) D.S. Conant., C. boliviana R. M. Tryon, es sinónimo de $C$. ruiziana, C. caracasana (H. Karst.) D.S. Conant, a lo largo de estos largos años ha sido confundida con especies como C. squamipes, $C$. herzogii Rosenst., C.carolihenrici, C. catacampta,- -cualquiera de las especies citadas en éste párrafo pero no C.caracasana.

C.caracasana var. boliviensis (Rosenst.) R.M. Tryon, los estudios taxonómicos actuales indican que es C. squamipes y a lo largo de los años la han identificado erróneamente (www.tropicos.org).

En el presente estudio determinamos a $C$. delgadii aff, como una especie afín pues las características morfológicas y ecológicas no se ajustan a C. delgadii.

Los resultados de la diversidad baja $\left(\mathrm{H}^{\prime}=1.57\right)$ y media $(1-\mathrm{D}=0.67)$ concuerda con la afirmación de Tejedor (2017) quien nos indica que la riqueza de especies de helechos arborescentes disminuye- 
-notablemente al incrementarse la distancia desde el ecuador. Tejedor (2017) indica asimismo, que la latitud tiene un fuerte efecto pues las comunidades en el Norte de Perú son casi el doble (10-15 especies) en comparación con las comunidades del sur de Perú (4 - 9 especies).

\section{De la estructura}

D. sellowiana, es la especie que presenta mayor número de individuos (Anexo 1 ) constituyendo un $50.76 \%$ de la población inventariada. Este alto porcentaje (Tab. 4) creemos se debe porque está dentro del rango distribución de la especie mencionada que va desde los 1700 a $3200 \mathrm{msnm}$. Y seguida de $L$. quadripinnata, que representa el $23.07 \%$. Este porcentaje también es lógico ya que está dentro de su amplia distribución de elevación que va entre 400- 3600 msnm. Las bajas abundancias de las especies (Tabla 2) C. delgadii aff. (4.61\%), C. squamipes, C. ruiziana $(3.07 \%)$ y $C$. carolihenrici, C. catacampta (1.53\%) se podría deber a el lugar de evaluación (2653 - 2800 msnm.) está al límite de sus rangos de elevación de las mismas (Tabla 3).

Tabla 3. Rango de elevación de helechos arborescentes. Tejedor (2017)

\begin{tabular}{|l|l|l|}
\hline Categoría & Escala & $\mathbf{N}^{\circ}$ Individuos \\
\hline Raro & 1 & $1-2$ individuos \\
\hline Infrecuente & 2 & $3-10$ individuos \\
\hline Frecuente & 3 & $11-20$ individuos \\
\hline Común & 4 & 2140 individuos \\
\hline Abundante & 5 & $>40$ individuos \\
\hline
\end{tabular}

D. sellowiana presentó una densidad de 50.76) y L. quadripinnata (23.07), a lo afirmado por Moreno etal. (2011) que indica que -
-D. sellowiana puede preferir ambientes con alta concentración de humedad relativa y suelos ricos en materia orgánica y respecto a $L$. quadripinnata Moreno et al. (2011) indica que es una especie que muchas veces establece competencia con D. sellowiana.

Como indica la Tabla 2 que D. sellowiana es la especie que tiene la mayor dominancia (83.84) en el área evaluada (Tab. 2). D. sellowiana es una especie que naturalmente es "grande y robusta" puede llegar a alcanzar los $10 \mathrm{~m}$. En la zona muestreada llegamos a evaluar individuos de hasta $9 \mathrm{~m}$ de alto y $120 \mathrm{~cm}$ de DAP.

\section{Índice de Valor de Importancia (IVI)}

D. sellowiana es la especie más importante (143.70), seguida de L. quadripinnata (36.78) que como lo habíamos indicado, son especies con un alto rango de distribución en elevación. Moreno, et al (2011) indica que D. sellowiana en Colombia, se desarrolla en áreas pequeñas sin ocurrencia homogénea y continua y en zonas cercanas a arroyos permanentes o zonas muy húmedas de bosques bien conservados. Montovani (2004) indica que las poblaciones de D. sellowiana en los bosques de Florianópolis - Brasil, también prefieren hábitat con alta humedad en el suelo y la atmósfera, y concentraciones elevadas de materia orgánica. El sector entre Wiñaywayna -Intipunku. también presenta dichas condiciones de conservación, alta humedad relativa y suelos húmedos. Los altos valores IVI de D. sellowiana y L. quadripinnata nos indican que la zona en estudio presenta un buen estado de conservación. Sobre todo D. sellowiana no crece en bosques disturbados.

Chávez (1992) reporta que la especie con mayor frecuencia, abundancia, densidad y dominancia es C.caracasana con 258 individuos- 
-por hectárea en Cedropata y 158 individuos por hectárea en Wiñay Wayna. Sin embargo, no podemos discutir con estos resultados pues ya sabemos que la distribución de C. caracasana no llega a Perú.Y en realidad se podría tratar de alrededor de 6 especies $(C$. squamipes, C. herzogii Rosenst., C.carolihenrici, C. catacampta, C.tungurague y C.cystolepis)

\section{Conclusiones}

1. El alto IVI y mayor abundancia de $D$. sellowiana sustenta que el bosque de la zona en estudio presenta un buen estado de conservación. Lo cual es muy importante por ser un grupo que está dentro de la lista CITES, apéndice II.

2. Para el sector de Wiñaywayna-Intipuncu se registró un total de 65 individuos, pertenecientes a 11 especies de helechos arborescentes: C. carolihenrici, C. catacampta, C. delgadii, C. ruiziana, C. squamipes, Cyathea sp1, Cyathea sp2, Cyathea sp3, Cyathea sp4, D. sellowiana, $L$. quadripinnata y la especie $C$. conjugata encontrada fuera del transecto de estudio.

3. C. carasana es una especie con distribución hacia el norte (a partir de Colombia, Venezuela, República Dominicana, Jamaica, Puerto Rico y Cuba como indica) y, no existe para Cusco.

4. Los resultados de la diversidad (Tab. 3) baja $\left(\mathrm{H}^{\prime}=1.57\right)$ y media $(1-\mathrm{D}=0.67)$ y riqueza media de especies, concuerda con la afirmación de Tejedor (2017) quien nos indica que la riqueza de especies de helechos arborescentes disminuye notablemente al incrementarse la distancia desde el ecuador, es decir, que la latitud tiene un fuerte efecto en las comunidades de helechos arborescentes.

5. Las altas densidades de $D$. sellowiana (50.76) y L. quadripinnata (23.07) se deberían a que ésta especie prefiere ambientes con alta- -concentración de humedad relativa presente en la zona evaluada. L. quadripinnata es una especie que en la zona de estudio también estableció competencia con $D$. sellowiana.

6. D. sellowiana, también es la especie que tiene la mayor dominancia (83.84) en el área evaluada (Tab. 3). D. sellowiana es una especie que naturalmente es "grande y robusta" puede llegar a alcanzar los $10 \mathrm{~m}$. En la zona muestreada llegamos a evaluar individuos de hasta $9 \mathrm{~m}$ de alto y $120 \mathrm{~cm}$ de DAP.

7. D. sellowiana es la especie con mayor importancia ecológica en el área evaluada (143.7\%), bajo condiciones de un hábitat continuo, conservado y húmedo.

\section{Agradecimientos}

$\mathrm{Al}$ vice-rectorado de investigación por el financiamiento. Al SERNAMP por las facilidades prestadas. Al Herbario CUZ de la Facultad de Ciencias, Escuela de Biología. A la Blga. Eufemia Machaca por las facilidades brindadas en la Estación Biológica de Wiñay Wayna. Al PhD Adrian Tejedor por su apoyo en la confirmación e identificación de las muestras. Al M.Sc. Abel Monteagudo M. por su ayuda y sugerencias. A la Blga. Heydy Borda Tamayo, M. Sc. Molleapaza Ugarte Susana, Blgo. Yuca Rivas Raul, Blga. Gladis Huallparimachi Quispe, Flores Huisa Karina, Espirilla Hancco Winnie y Huaman Ovalle Naysha.

\section{Literatura citada}

Chávez, H.W.1992. “Estudio Poblacional De Helechos Arborescentes EnWiñay Wayna (Santuario Histórico De Machupicchu) Y Cedropata”. Seminario Curricular, Facultad De Ciencias Biológicas, UNSAAC. 
Galiano, W. y Nuñez, M. P. (2011). Sinopsis de las especies arbóreas del Santuario Histórico de Machupicchu (SHM) y su Zona de Amortiguamiento (ZA). Revista El Antoniano $N^{\circ}$ 117: 178-194. UNSAAC-DCPS. Cusco - Perú.

Galiano, S. W., Tupayachi, H.A., Farfán, V.J., Huamán, O. D., Moreno, H. M., Tupayachi,T. R., Suclli, M. E., Carazas,M. F., Monteagudo, M. A., Nuñez,V.P, Valenzuela,G. L. (2002) Ampliación del inventario de Biodiversidad Botánica en el Santuario Histórico de Machu Picchu. Universidad Nacional de San Antonio Abad del Cusco. PROFONAMPE. Programa Machu Picchu. Informe in extenso.

Holdridge, L. R. (1967). «Life Zone Ecology». Tropical Science Center. San José, Costa Rica. (Traducción del inglés por Humberto Jiménez Saa: «Ecología Basada en Zonas de Vida», 1ra. ed. San José, Costa Rica: IICA, 1982).Kramer K.U. (1990). Dicksoniaceae. The Families and genera of vascular plants. Springer-Verlag. Berlin.

Krebs C.J. 1978. Ecology: The Experimental Analysis of Distribution and Abundance. 2nd Edition. Haper \& Row, Publishers, INC. New York, USA.

Lehnert, M. (2009) Resolving the Cyathea caracasana complex ()polypodiopsida: Cyatheaceae. Stuttgarter Beitrage zur Naturkunde A, Neue Serie 2:409-445; Stuttgart, 30.IV.2009

Lehnert, M. ( 2011 ). The Cyatheaceae (Polypodiopsida) of Peru. Brittonia 63, 11-45.

Magurran, A.E. (2004) Measuring Biological Diversity. Blackwell Publishing, Oxford, 256 p.

Macedo, L. (2013). Composición florística, índice de valor de importancia y volumen maderable de especies comerciales de un bosque natural de colina baja, distrito de Pevas (Tesis de pregrado). Universidad Nacional de la Amazonia Peruana, Loreto, Perú.

Mantovani, M. (2004) Caracterização de populações naturais de Xaxim (Dicksonia sellowiana (Presl.) Hooker, em diferentes condições edafo-climáticas no Estado de Santa Catarina: UFSC, 2004. 105p. Dissertação (Mestrado em Recursos Genéticos Vegetais) - Universidade Federal de Santa Catarina, Florianópolis.

Matteucci S. \& Colma A. (1982). Metodologías para el Estudio de la Vegetación. Secretaria General de la Organización de los Estados Americanos. Washington DC, USA.

Rev. Q'EUÑA 10 (1): 45 (Agosto 2019)

Sociedad Botánica del Cusco
Moreno, C. E. (2001). Métodos para Medir la Biodiversidad.Volumen I. Manuales y Tesis. Socie-dad Entomológica Aragonesa. Zaragoza, España. 84 pp.

Moreno, A. R. A., Cadena V., C. E., Morales, G., Peña, N y Pérez, B. (2011) Conservación Integral de Dicksonia sellowiana Hook., en Bogotá D.C. y su área de influencia. Rev. Acad. Colomb. Cienc: Volumen XXXV 134 marzo.

Mostacedo B. \& Fredericksen T.S. (2000) Manual de Métodos Básicos de Muestreo y Análisis en Ecología Vegetal. Editora El País. Santa Cruz, Bolivia.

Oksanen J., Blanchet, F. G., Friendly, M., Kindt, R., Legendre, P., McGlinn, D., Peter Minchin, P. R., O'Hara, R. B., Simpson, G. L., Solymos, P., Stevens, M. H. H., Szoecs, E. and Wagner, H. (2019). vegan: Community Ecology Package. R package version 2.5-6. https://CRAN.Rproject.org $/$ package $=$ vegan

Ramírez-Barahona S.A., Luna-Vega I. y TejeroDíez D. (2011). Species richness, endemism, and conservation of American tree ferns (Cyatheales). Biodiversity and Conservation.

Rojas, A. (1999). Helechos arborescentes de Costa Rica. Santo Domingo, Heredia, Costa Rica, INBIO. 174 pp.

R Core Team (2020). R: A language and environment for statistical computing. R Foundation for Statistical Computing, Vienna, Austria. URL https: / / www.Rproject.org/.

Tejedor, A. (2017). Diversity and endemism of tree ferns (Cyatheaceae: Polipodiopsida) in the Central Andes along latitudinal and elevation gradients. Acta Botanica Malacitana 42, Núm. 1, 41-47 DOI: http;/dx.doi.org/10.24310/abm.v42i1.2959

Tuomisto, H.,Ruokolainen, K., Poulsen, A.D., Moran, R. C., Quintana, C., Cañas, G., Celi, J. (2002) Distribution and Diversity of pteridophytes and Melastomataceaealong Edaphic Gradients in Yasuni National Park, Ecuadorian Amazonia. Biotropica 34(4): 516-533.

Villarreal, H., Ávarez, M., Cordoba, S., Escobar, F., Fagua, G., Gast, F., Umaña, A. M. (2004). Manual de métodos para el desarrollo de inventarios de biodiversidad. Programa de Inventarios de Biodiversidad. Instituto de Investigación de Recursos Biológicos Alexander von Humboldt. Bogotá, Colombia, 236 p. 
Holgado et al: diversidad y composición de helechos arbóreos en Wiñayna-Intipunku (SHMP)

Anexo 1. Listado de las especies inventariadas y sus parametros poblacionales

\begin{tabular}{|c|c|c|c|c|c|}
\hline $\begin{array}{l}\text { Unidad de } \\
\text { muestreo }\end{array}$ & $\mathbf{N}^{\circ}$ & Especies & $\begin{array}{l}\text { Altura } \\
\text { (m) }\end{array}$ & $\begin{array}{l}\text { DAP } \\
(\mathrm{cm})\end{array}$ & $\begin{array}{l}\text { Altura } \\
\text { parcela }\end{array}$ \\
\hline \multirow[t]{3}{*}{1} & 1 & Cyathea squamipes $\mathrm{H}$. Karst. & 0.7 & 7 & 18 \\
\hline & 2 & Cyathea squamipes H. Karst. & 0.7 & 3 & 41 \\
\hline & 3 & Cyathea sp3 & 0.7 & 10 & 50 \\
\hline \multirow[t]{4}{*}{2} & 4 & Cyathea delgadii aff. & 1.5 & 33 & 10 \\
\hline & 5 & Lophosoria quadripinnata (J.F. Gmel.) C. Chr. & & 14 & \\
\hline & 6 & Cyathea delgadii aff. & 5 & & 15 \\
\hline & 7 & Cyathea delgadii aff. & 8 & 70 & 22 \\
\hline \multicolumn{6}{|l|}{3} \\
\hline \multirow[t]{8}{*}{4} & 8 & Cyathea carolihenrici Lehnert & 8 & 15 & 16 \\
\hline & 9 & Dicksonia sellowiana Hook. & 8 & 56 & 21 \\
\hline & 10 & Dicksonia sellowiana Hook. & & 51 & 25 \\
\hline & 11 & Dicksonia sellowiana Hook. & 0.7 & 35 & 35 \\
\hline & 12 & Dicksonia sellowiana Hook. & 3 & 75 & 35 \\
\hline & 13 & Lophosoria quadripinnata (J.F. Gmel.) C. Chr. & 0.2 & 39 & 37 \\
\hline & 14 & Lophosoria quadripinnata (J.F. Gmel.) C. Chr. & 0.3 & 23 & 39 \\
\hline & 15 & Lophosoria quadripinnata (J.F. Gmel.) C. Chr. & 0.18 & 19 & 39 \\
\hline \multirow[t]{18}{*}{5} & 16 & Lophosoria quadripinnata (J.F. Gmel.) C. Chr. & 23 & 8 & \\
\hline & 17 & Lophosoria quadripinnata (J.F. Gmel.) C. Chr. & & 9 & \\
\hline & 18 & Dicksonia sellowiana Hook. & 9 & 120 & 10 \\
\hline & 19 & Dicksonia sellowiana Hook. & 2 & 51 & 15 \\
\hline & 20 & Cyathea sp1 & 0.25 & 22 & 17 \\
\hline & 21 & Dicksonia sellowiana Hook. & 0.12 & 10 & 17 \\
\hline & 22 & Dicksonia sellowiana Hook. & 0.2 & 12 & 16 \\
\hline & 23 & Dicksonia sellowiana Hook. & 6.5 & 124 & 21 \\
\hline & 24 & Cyathea spl & 0.1 & 12 & 25 \\
\hline & 25 & Dicksonia sellowiana Hook. & 1 & & 28 \\
\hline & 26 & Dicksonia sellowiana Hook. & 2 & 47 & 32 \\
\hline & 27 & Cyathea catacampta Alston & 4 & 35 & 37 \\
\hline & 28 & Cyathea spl & & & 34 \\
\hline & 29 & Cyathea sp4 & 0.4 & 45 & 37 \\
\hline & 30 & Cyathea ruiziana Klotzsch & 8 & 71 & 47 \\
\hline & 31 & Lophosoria quadripinnata (J.F. Gmel.) C. Chr. & & 50 & \\
\hline & 32 & Cyathea ruiziana Klotzsch & 8 & 80 & 48 \\
\hline & 33 & Cyathea spl & 10 & 12 & 50 \\
\hline \multirow[t]{2}{*}{6} & 34 & Lophosoria quadripinnata (J.F. Gmel.) C. Chr. & 0.1 & 8 & 23 \\
\hline & 35 & Dicksonia sellowiana Hook. & 1 & 44 & 44 \\
\hline
\end{tabular}


Holgado et al: diversidad y composición de helechos arbóreos en Wiñayna-Intipunku (SHMP)

\begin{tabular}{|c|c|c|c|c|c|}
\hline \multirow[t]{11}{*}{7} & 36 & Lophosoria quadripinnata (J.F. Gmel.) C. Chr. & 0.1 & 30 & 0 \\
\hline & 37 & Lophosoria quadripinnata (J.F. Gmel.) C. Chr. & 0.2 & 25 & 2 \\
\hline & 38 & Dicksonia sellowiana Hook. & 2 & 45 & 6 \\
\hline & 39 & Dicksonia sellowiana Hook. & 5 & 70 & 14 \\
\hline & 40 & Lophosoria quadripinnata (J.F. Gmel.) C. Chr. & 0.16 & 15 & 16 \\
\hline & 41 & Dicksonia sellowiana Hook. & 5.5 & 64 & 20 \\
\hline & 42 & Dicksonia sellowiana Hook. & 8 & 30 & 23 \\
\hline & 43 & Dicksonia sellowiana Hook. & 7 & 186 & 33 \\
\hline & 44 & Dicksonia sellowiana Hook. & 2 & 38 & 39 \\
\hline & 45 & Dicksonia sellowiana Hook. & 1 & 50 & 42 \\
\hline & 46 & Dicksonia sellowiana Hook. & 0.5 & 22 & 43 \\
\hline \multirow[t]{2}{*}{8} & 47 & Cyathea sp2 & 1 & 33.8 & 0 \\
\hline & 48 & Dicksonia sellowiana Hook. & 2.5 & 76 & 5 \\
\hline \multirow[t]{4}{*}{9} & 49 & Dicksonia sellowiana Hook. & 1 & & 1 \\
\hline & 50 & Lophosoria quadripinnata (J.F. Gmel.) C. Chr. & & 1 & \\
\hline & 51 & Lophosoria quadripinnata (J.F. Gmel.) C. Chr. & 4 & 40 & 23 \\
\hline & 52 & Dicksonia sellowiana Hook. & 9 & 170 & 23 \\
\hline \multirow[t]{13}{*}{10} & 53 & Dicksonia sellowiana Hook. & 5.5 & & 1.3 \\
\hline & 54 & Dicksonia sellowiana Hook. & 2.15 & 78 & 7 \\
\hline & 55 & Dicksonia sellowiana Hook. & 4 & 110 & 9 \\
\hline & 56 & Dicksonia sellowiana Hook. & 5 & 60 & 10 \\
\hline & 57 & Dicksonia sellowiana Hook. & 0.15 & 12 & 15 \\
\hline & 58 & Dicksonia sellowiana Hook. & 0.15 & 9 & 15 \\
\hline & 59 & Dicksonia sellowiana Hook. & & 70 & 18.5 \\
\hline & 60 & Dicksonia sellowiana Hook. & 3 & 60 & 22 \\
\hline & 61 & Dicksonia sellowiana Hook. & 2 & 80 & 23 \\
\hline & 62 & Cyathea sp2 & 0.9 & 26 & 24 \\
\hline & 63 & Lophosoria quadripinnata (J.F. Gmel.) C. Chr. & 1 & 47 & 25 \\
\hline & 64 & Lophosoria quadripinnata (J.F. Gmel.) C. Chr. & 0.5 & 30 & 31 \\
\hline & 65 & Dicksonia sellowiana Hook. & 4 & & 25 \\
\hline
\end{tabular}

